\title{
High-dimensional Quantum Dynamics Study on Excitation-Specific Surface Scattering including Lattice Effects of a Five-Atoms Surface Cell
}

\section{SUPPORTING INFORMATION}

Qingyong Meng, ${ }^{1, *}$ Markus Schröder, ${ }^{2, \dagger}$ and Hans-Dieter Meyer ${ }^{2, \dagger}$

${ }^{I}$ Department of Chemistry, Northwestern Polytechnical University,

West Youyi Road 127, 710072 Xi'an, China

${ }^{2}$ Theoretische Chemie, Physikalisch-Chemisches Institut,

Ruprecht-Karls Universität Heidelberg,

Im Neuenheimer Feld 229, D-69120 Heidelberg, Germany

(Dated: March 8, 2021)

*Electronic address: qingyong.meng@nwpu.edu.cn

†Electronic address: markus.schroeder@pci.uni-heidelberg.de

‡Electronic address: hans-dieter.meyer@pci.uni-heidelberg.de 


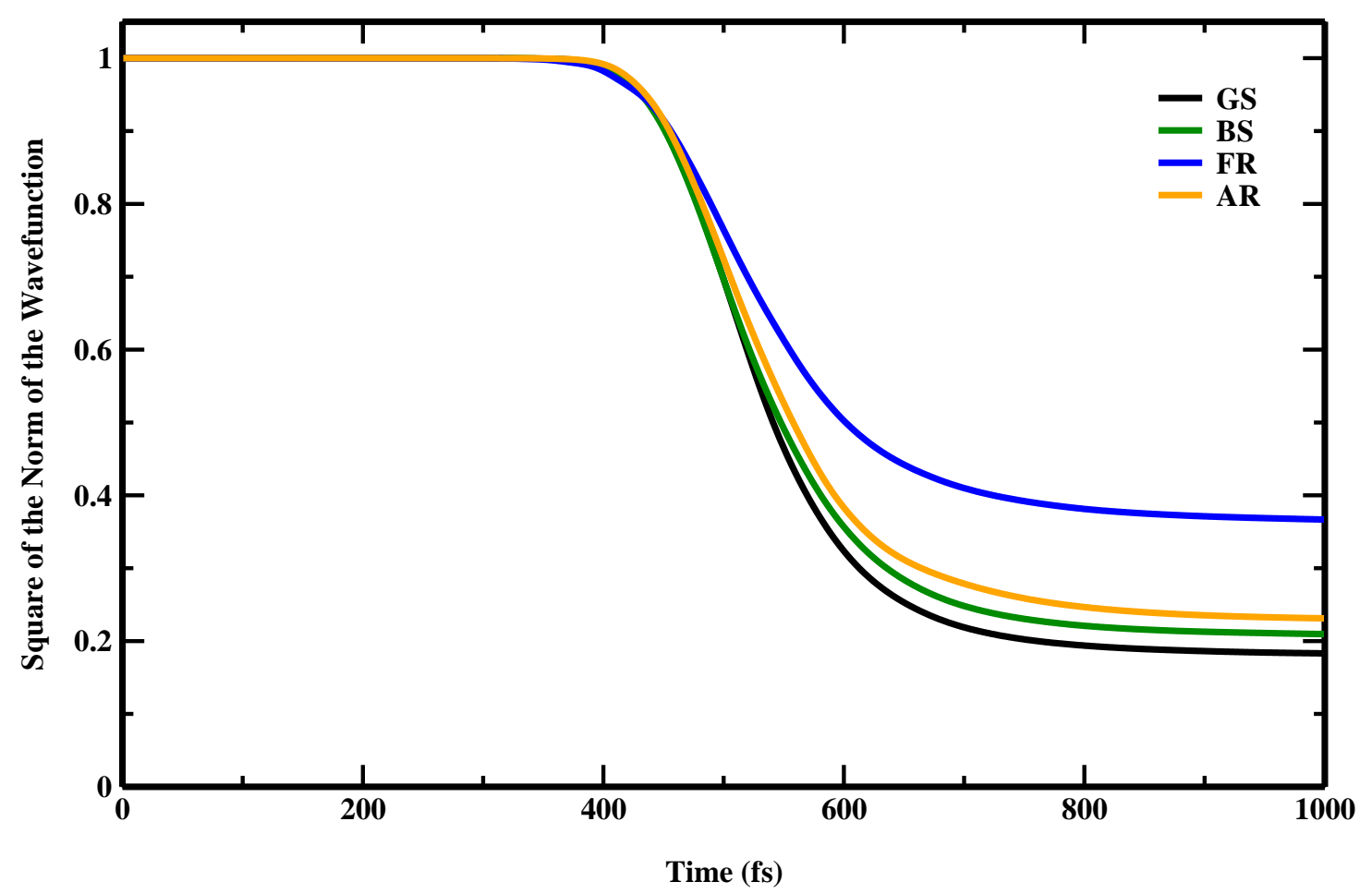

FIG. 1: Comparsion of the square of the wavefunction norm from the 6D model, where colored lines represent the results computed by various excited states and the the propagation time is cut at $1000 \mathrm{fs}$. 


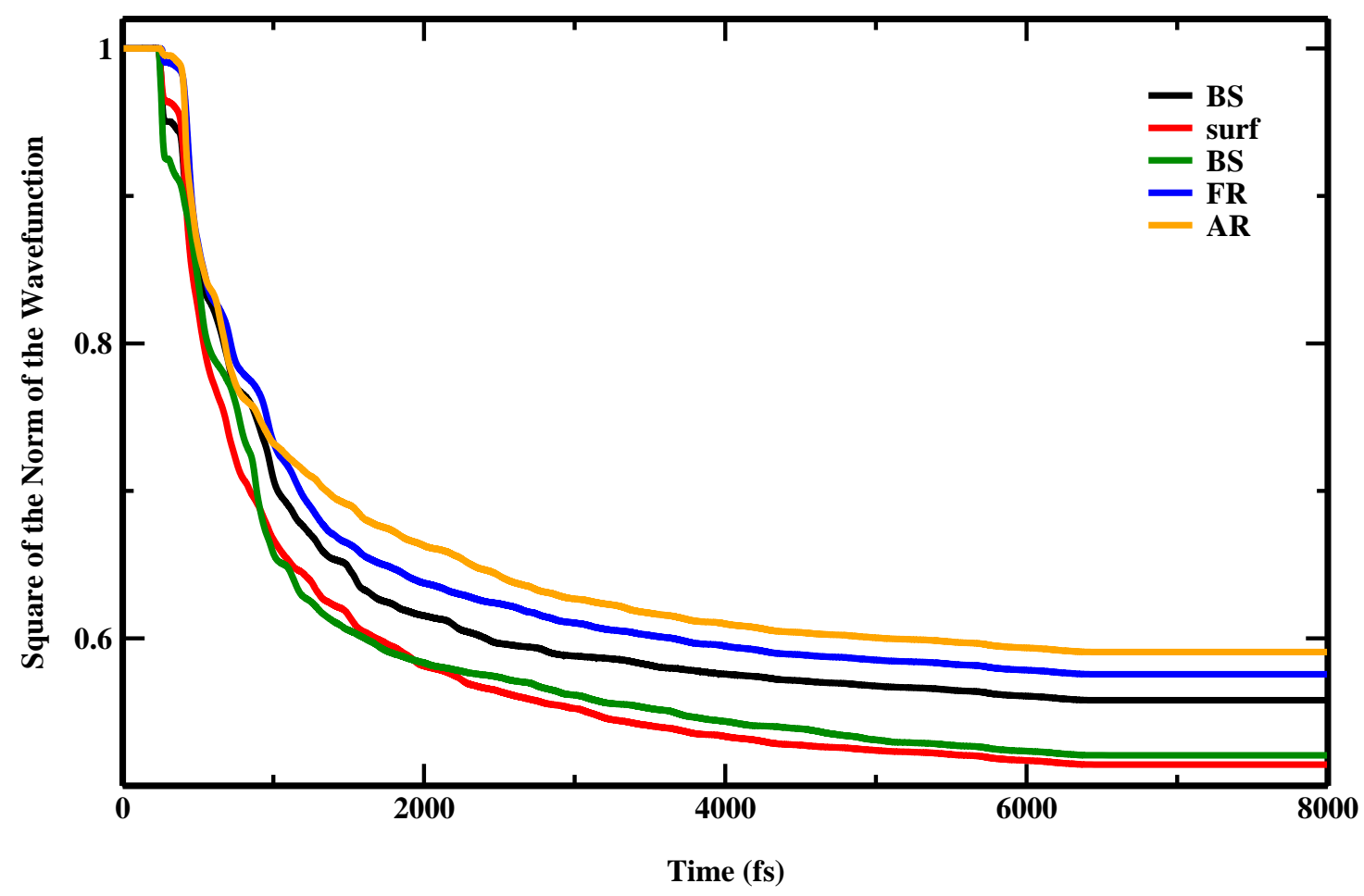

FIG. 2: Same as Figure 1, except for the results from the 21D model. 


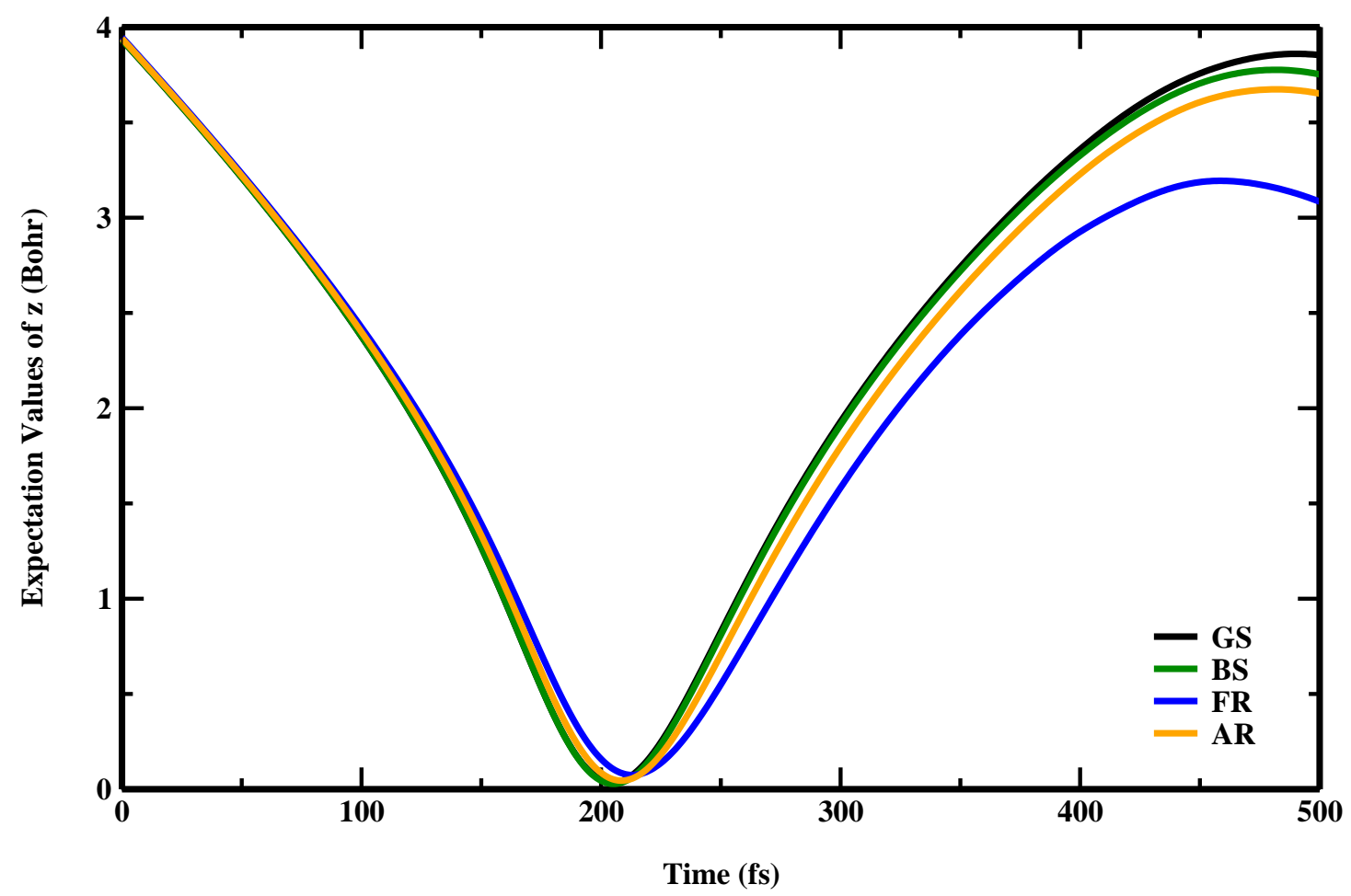

FIG. 3: Comparsion of the time-dependent position expectation values along the $z$ coordinate of CO from the 6D model, that is $\langle z(t)\rangle=\langle\psi(t)|z| \psi(t)\rangle$. The colored lines represent the results computed by various excited states and the the propagation time is cut at $500 \mathrm{fs}$, where the GS, BS, FR, and AR are presented by black, green, blue, and yellow lines, respectively. 


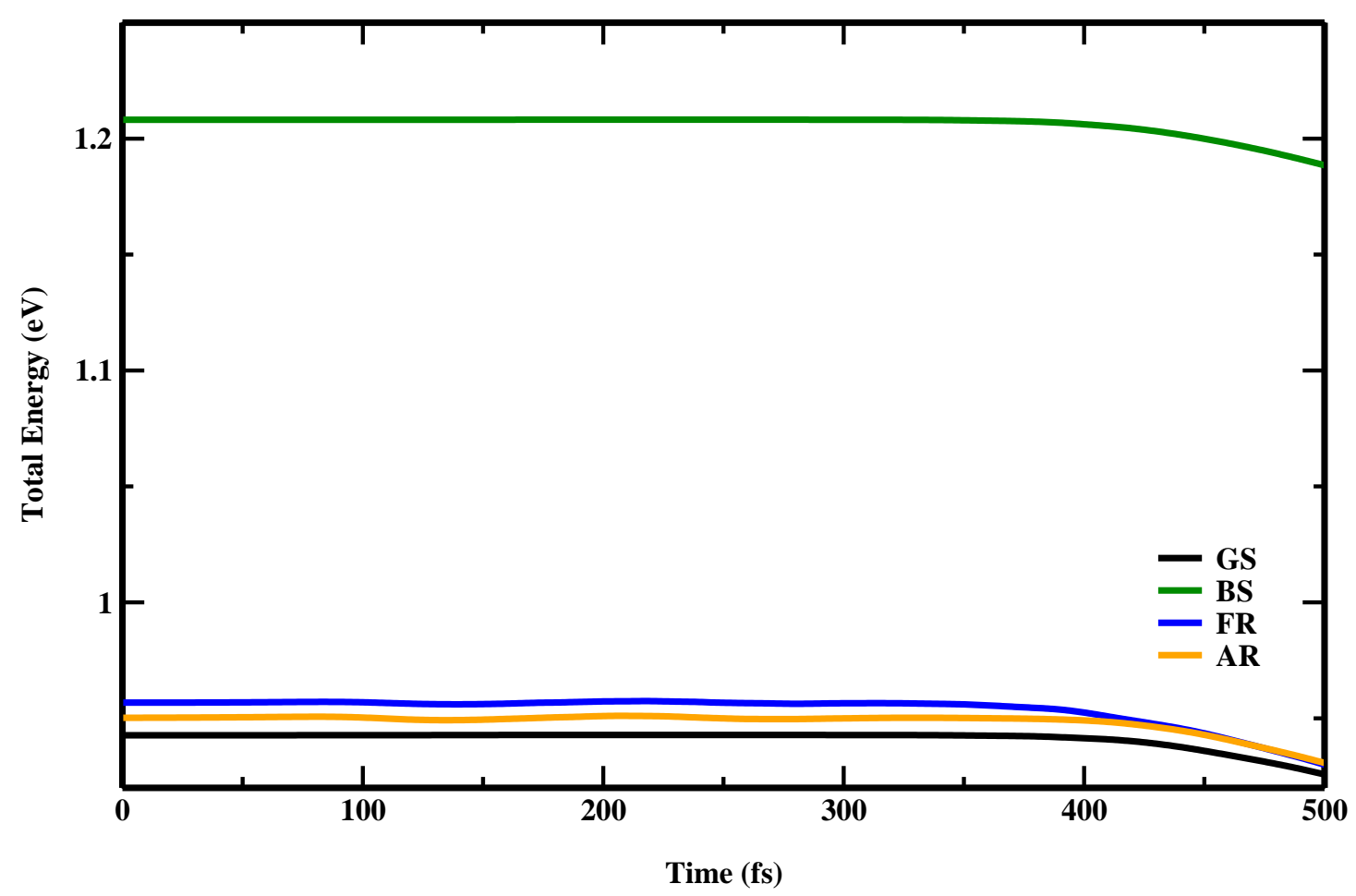

FIG. 4: Comparsion of the time-dependent expectation values of the total energy (i.e., kinetic energy plus potential energy) of $\mathrm{CO}$ from the 6D model, that is $\left\langle E_{\mathrm{CO}}(T)\right\rangle=\left\langle\psi(t)\left|H_{s}\right| \psi(t)\right\rangle$. The colored lines (see also the caption of Figure 3) represent the results computed by various excited states and the the propagation time is cut at $500 \mathrm{fs}$. 


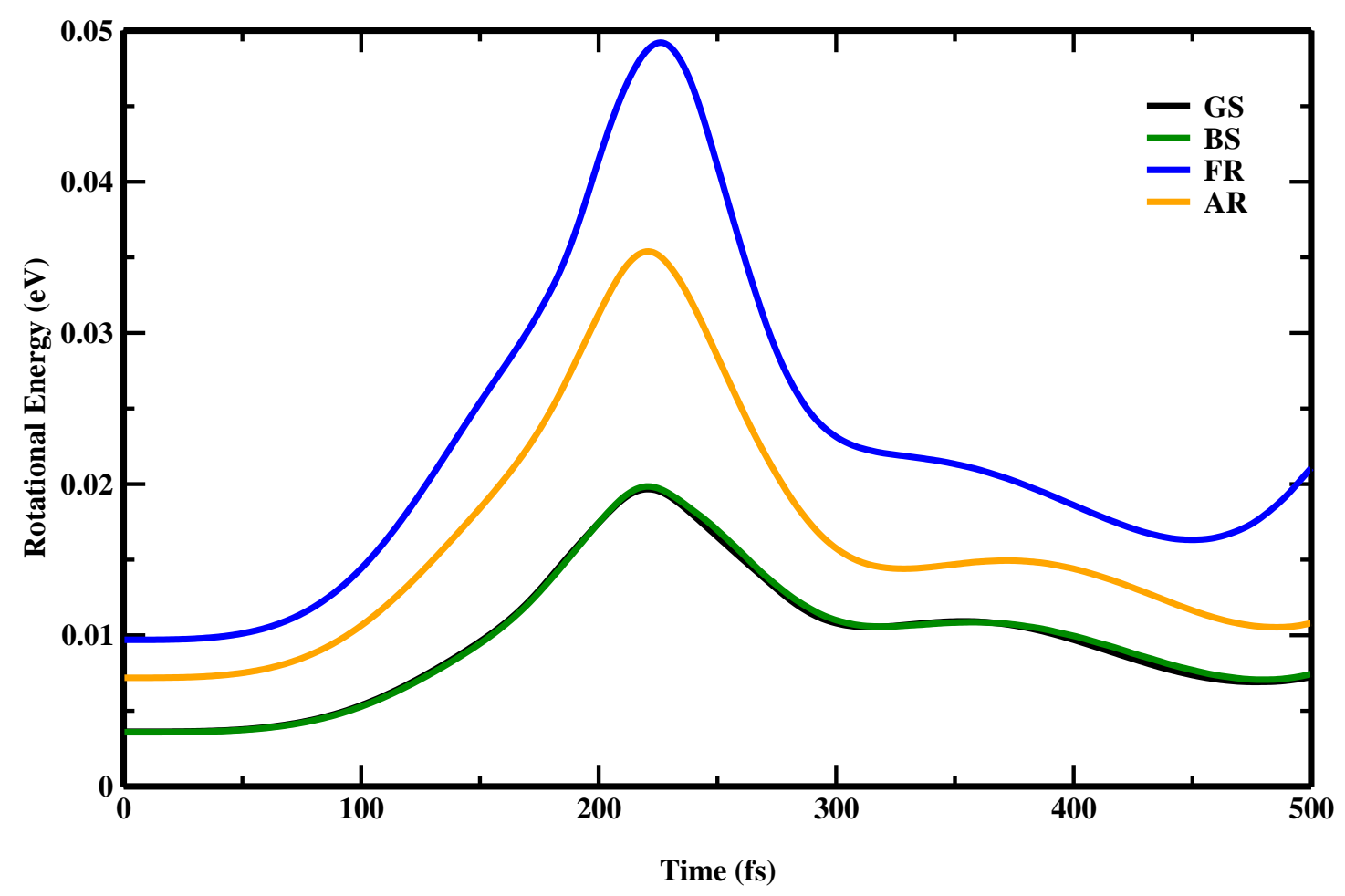

FIG. 5: Comparsion of the time-dependent expectation values of the rotational energy of CO from the 6D model, that is $\left\langle T_{\text {ang }}(t)\right\rangle=\left\langle\psi(t)\left|j^{2} /\left(2 \mu r^{2}\right)\right| \psi(t)\right\rangle$, where $j^{2}$ is the angle part of the Laplacian operator in spherical coordinates. The colored lines (see also the caption of Figure 3 ) represent the results computed by various excited states and the the propagation time is cut at $500 \mathrm{fs}$. 


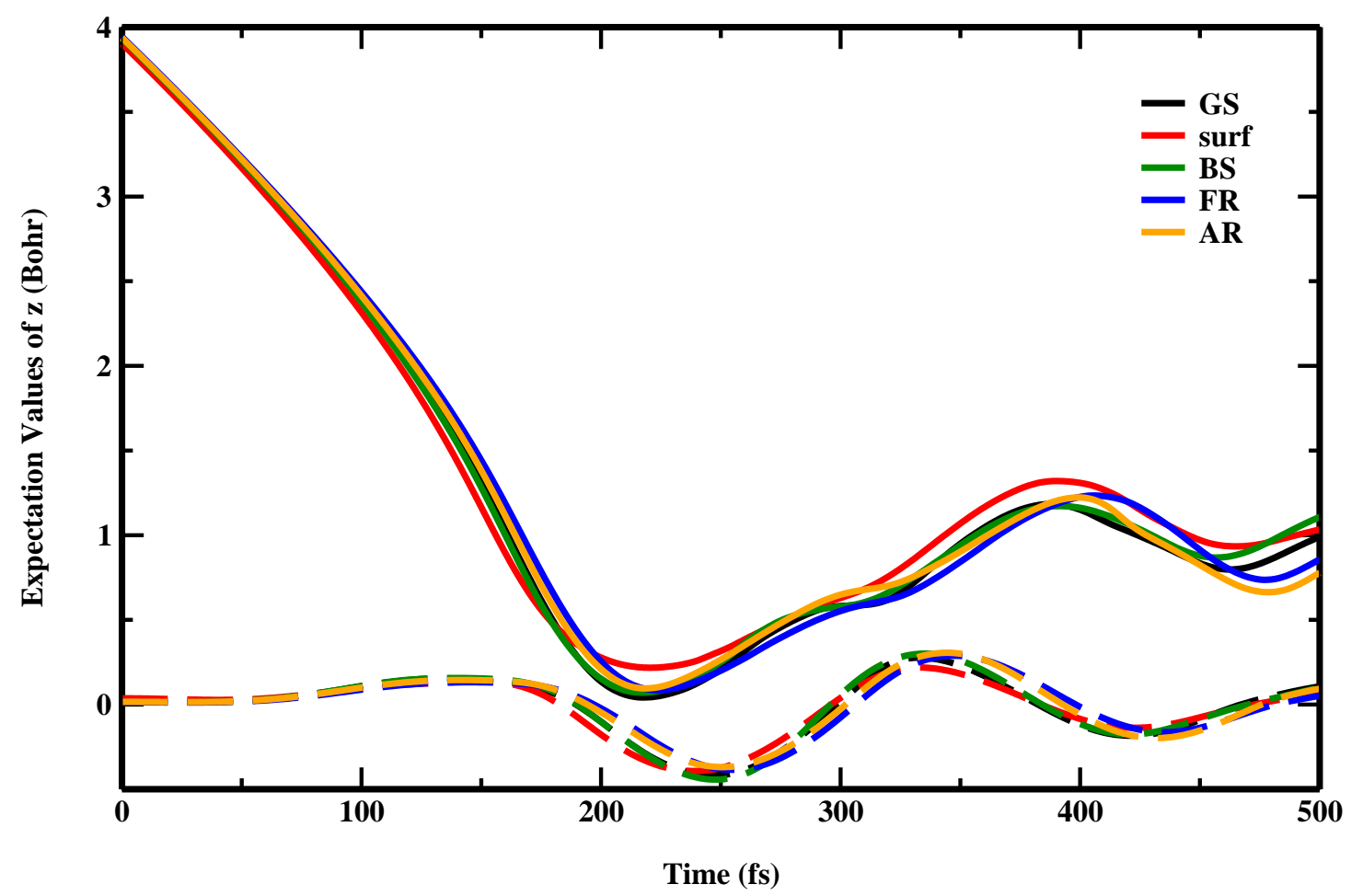

FIG. 6: Comparsion of the time-dependent position expectation values along the $z$ axis of both $\mathrm{CO}$ and the top atom from the 21D model, that is $\langle z(t)\rangle=\langle\psi(t)|z| \psi(t)\rangle$ and $\left\langle Q_{1 z}(t)\right\rangle=\left\langle\psi(t)\left|Q_{1 z}\right| \psi(t)\right\rangle$. The solid and dashed lines represent $\langle z(t)\rangle$ and $\left\langle Q_{1 z}(t)\right\rangle$, respectively. The colored lines represent the results computed by various excited states and the the propagation time is cut at $500 \mathrm{fs}$, where the GS, surf, BS, FR, and AR are presented by black, red, green, blue, and yellow lines, respectively. 


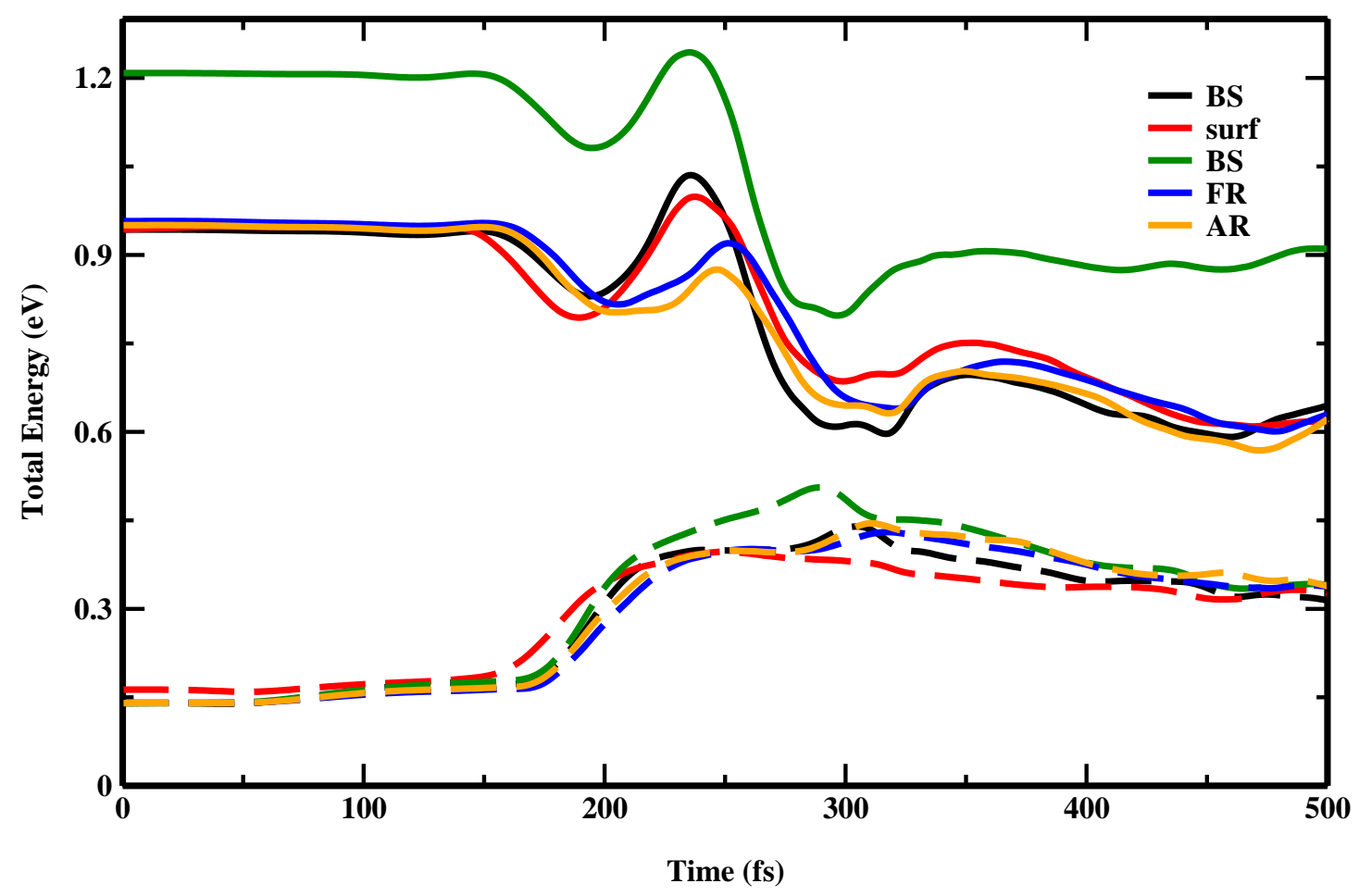

FIG. 7: Same as Figure 6, except for the comparsion of the time-dependent expectation values of the total energy of both $\mathrm{CO}$ and the $\mathrm{Cu}$ surfac from the 21D model, that is $\left\langle E_{\mathrm{CO}}(t)\right\rangle=\left\langle\psi(t)\left|H_{s}\right| \psi(t)\right\rangle$ and $\left\langle E_{\text {surf }}(t)\right\rangle=\left\langle\psi(t)\left|H_{b}\right| \psi(t)\right\rangle$. The solid and dashed lines represent $\left\langle E_{\mathrm{CO}}(t)\right\rangle$ and $\left\langle E_{\mathrm{surf}}(t)\right\rangle$, respectively. 


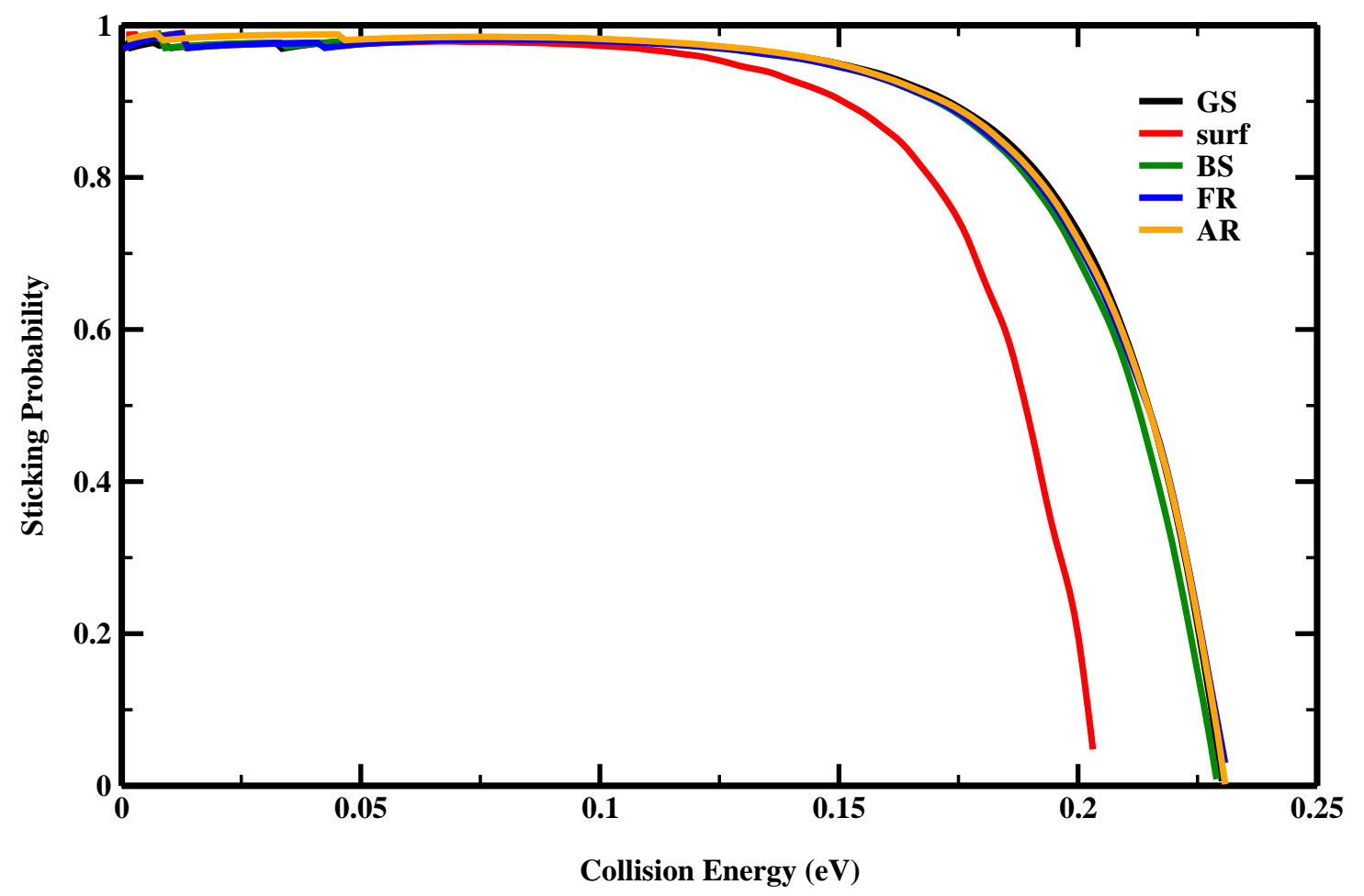

FIG. 8: Sticking probabilities versus collision energy (in $\mathrm{eV}$ ) computed for the 21D model with flexible surface atoms. The black and green lines represent the sticking probabilities when the $\mathrm{CO}$ molecule is initially in the ground state (denoted by GS) and vibrational excited state of the $\mathrm{CO}$ bond stretch mode (denoted by BS), the yellow and blue lines represent those where the molecule is in the excited states associated with frustrated azimuth angle $\phi$ (denoted by AR) and zenith angle $\theta$ (denoted by FR), respectively. The red line represents the sticking probability when the molecule is initially in the ground state but the surface is in the excited state associated with the out-of-plane mode of the center atom (denoted by surf). 